\title{
User Needs in Green ITS: Results of a Questionnaire Survey and Proposal for Green ITS Design
}

\author{
Wing Yan Man $\cdot$ Jing Bie $\cdot$ Bart van Arem
}

Received: 25 November 2010 /Revised: 7 July 2011 /Accepted: 4 August 2011 / Published online: 24 September 2011

(C) The Author(s) 2011. This article is published with open access at Springerlink.com

\begin{abstract}
An internet survey has been conducted among drivers in the Netherlands and Japan to study their attitude towards Green ITS. The survey focuses on driving behavior and ITS experience, attitude towards environment, and Green ITS preferences. The results show that money-related information gives the most effective motivation for drivers to follow fuel efficient behavior; the least preferred information is impact on environment. Correlations are found between information preference and the driving style. The majority of the respondents intend to make frequent use of Green ITS. Based on these results, a new design for Green ITS is proposed, providing drivers with information on driving behavior, expenses on fuel consumption and environmental factors.
\end{abstract}

Keywords Green ITS · User needs · Fuel efficiency · Questionnaire survey $\cdot$ User interface

W. Y. Man

Department of Civil Engineering, Faculty of Engineering

Technology, University of Twente,

Drienerlolaan 5,

7522 NB Enschede, The Netherlands

e-mail: w.y.man@student.utwente.nl

J. Bie $(\bowtie)$

Centre for Transport Studies, Faculty of Engineering Technology,

University of Twente,

Drienerlolaan 5,

7522 NB Enschede, The Netherlands

e-mail: j.bie@utwente.nl

B. van Arem

Department of Transport and Planning, Faculty of Civil

Engineering and Geosciences, Delft University of Technology,

$2628 \mathrm{CN}$ Delft, The Netherlands

e-mail: b.vanarem@tudelft.nl

\section{Introduction}

Excessive energy consumption and greenhouse gas (GHG) emissions are making changes to our living environment that will be damaging in the long term. A prominent example is global warming, as acknowledged in the Kyoto Protocol [1]. Road traffic has been a major contributor to such changes. Now, more than ever, people are being encouraged to act upon it to create a better environment for the future. Diminishing car use would decrease GHG productions [2] but this is difficult to achieve in this modern and dynamic world [3]. A realistic solution would be to improve fuel efficiency, such as to guide our drivers to a fuel efficient driving behavior. High speeds, heavy acceleration and lack of anticipation in traffic have been identified as the main causes of excessive emission production [4]. By improving the driving skills to a more environmentally friendly style, we can reduce fuel consumption, fuel expenses as well as emission production. This benefits both the individual and the environment.

Green ITS (Green Intelligent Transport System) monitors the driver's performance in order to achieve a more fuel efficient behavior. By displaying information on driving history and $\mathrm{CO}_{2}$ emission, the system informs drivers of their impact on the environment. Nowadays, there are a few systems available on the market that monitor driving behavior, such as Fiat eco:Drive [5] which reports on the fuel economy after the trip. In other systems, fuel consumptions are shown to the driver dynamically. To the best of our knowledge, no user feedback has been published in terms of driver response and behavioral adaptation due to Green ITS. To promote and improve these systems, the design and functioning should satisfy the desires of the user [6]. By measuring the attitude of drivers towards Green ITS, designers will be aware of the needs 
and preferences of drivers. Green ITS can then be designed from both a technological point of view and the preferences of the driver.

This research focuses on user needs in Green ITS. The objective is to improve current designs of Green ITS by considering factors that motivate drivers to change their behavior. Based on the results of an internet survey, this paper will elaborate on user attitude and preferences towards Green ITS among Dutch and Japanese drivers. A new design of Green ITS is then proposed which motivates drivers to follow environmentally friendly driving behavior.

\section{Background}

To achieve sustainable mobility, various measures are being implemented to reduce GHG emissions around the globe. Traditional measures include increasing capacity of the road network (such as constructing extra lanes) and imposing fuel tax for transport [7]. The car industry has been active in introducing electric/hybrid cars and information technology systems that support the driving task [8]. For reducing GHG emission, researchers have been focusing on engine technologies, policy measures, changes to the infrastructure, modal shift, transport management, and driving behavioral changes.

In this paper we focus on the driving behavioral changes. For most drivers it is not easy to change their current driving behavior by adopting new skills. To help the driver in changing his (or her) behavior, in-car Green ITS systems and services are being developed. These systems aim at achieving a fuel efficient behavior. They provide the driver with information on his driving behavior: the driver is made known of his performance by several indicators, displayed during and/or after the trip. Examples of the indicators include characteristics of fuel consumption and speed profile. More advanced systems collect driver behavior data from previous trips and compare them with the optimal fuel efficient behavior. Personal advices can then be provided to the driver to achieve the optimal fuel efficiency. It is also possible that these advices are given in real time (i.e. during the trip, while the driver is driving). Such a system monitors the current driving behavior and intervenes if inefficient behavior is being practiced. The driver receives direct feedbacks on his behavior (progression) and advices on changing his behavior for better fuel efficiency.

\section{Questionnaire Survey}

For a successful design of in-car Green ITS systems and services, user needs and acceptance are essential. We have deployed a questionnaire survey to investigate drivers' opinions towards Green ITS. The purpose of this survey is to assess the following aspects:

- Type of preferred information for motivating the driver to change into fuel efficient behavior;

- Influence of driver's personal characteristics on the type of preferred information;

- Attitude towards Green ITS.

The currently available in-car technologies are mostly designed from a technological point of view. With the knowledge of driver attitude and preferences, we can then improve the current designs of Green ITS.

\subsection{Questionnaire Design}

The questionnaire survey "Green light for a better environment" has been deployed and it consists of questions on the following four subjects:

- General information;

- Driving behavior \& ITS experience;

- Attitude towards environment;

- Attitude towards Green ITS.

The survey first provides the respondent with a brief introduction on Green ITS. This introduction does not include the detailed attributes of Green ITS (such as the possibility of different interfaces, sounds and lights), in order to avoid confusion and pre-selection bias.

General Information This part consists of questions on the personal characteristics of the respondent. Only respondents possessing a driving license are asked to participate in the survey. The questions are related to age, occupation, type of driving licenses, vehicle ownership, and vehicle usage (frequency and vehicle features). With this information drivers can be categorized. Factors such as driving experience and personality can also be easily retrieved from the dataset.

Driving Behavior \& ITS Experience Questions in this part are related to average mileage, driving frequency, trip purposes, route choice, and familiarity with ITS. By investigating the respondent's driving behavior we can identify which driver group yields the highest impact on the environment. This driver group needs special support from Green ITS. Also, driver experience with ITS is studied to assess driver familiarity with ITS technologies, which influences driver adaptability towards Green ITS. This is an important factor in the design of Green ITS.

Attitude Towards Environment Drivers are asked to rank several types of information in terms of the likelihood to 
trigger a behavioral change on them. Other questions relate to the attitude towards environment in general and the self image of the respondent on environmental friendliness. By measuring driver attitude towards the environment we can identify the type of drivers who have a positive attitude and are therefore more willing to change their behavior for a better environment. Drivers who are not impressed by the environmental issues need other stimulus for changing their behavior.

Attitude Towards Green ITS To design a Green ITS system that satisfies the desires of the driver, we need to measure the driver's attitude. Many drivers are not yet familiar with in-car Green ITS, because it is still a novelty. To measure their attitude towards such systems, examples of in-car Green ITS are provided to give the respondents a reasonable impression. Respondents are then asked which type of information is relevant to help them in adapting their behavior. Four categories of information are considered:

- general information such as fuel consumption, costs and $\mathrm{CO}_{2}$ emission;

- advice on driving behavior;

- analysis of previous driving behavior;

- progress in driving behavior.

Besides the type of information, the timing of information provision is also studied.

\subsection{Conducting the Survey}

Internet survey has been chosen as survey method. It has the advantages of easy accessibility, low costs, direct collection of digital data, and convenience in data processing. Internet surveys provide an effective way of reaching the drivers and collecting their opinions. In this study we only consider drivers from the Netherlands and Japan. As of June $2010,88.3 \%$ of the whole population in the Netherlands are internet users. The same statistic is $78.2 \%$ for Japan [9]. We expect the ratio of internet users among Dutch and Japanese drivers is around or even higher than these percentages. Therefore internet surveys do not suffer greatly from the selection bias of excluding non-internet users.

The survey is set up in both the Dutch and Japanese languages. Limeservice (www.limeservice.com) has been chosen as the survey interface owing to its ability to edit both Latin and Japanese characters. The final web survey is hosted on the following web pages:

Dutch version: http://wingyan.limeask.com/index.php? sid $=56984 \&$ lang $=\mathrm{nl}$

Japanese version: http://wingyan.limeask.com/index. php?sid=97472\&lang $=$ ja
The survey was conducted in the period between December 2008 and February 2009. The respondents were informed of the survey by an email which included information on the research and a link to the survey webpage. Respondents in the Netherlands were reached through a personal network. They were also asked to forward the questionnaire among their social networks. To reach the Japanese respondents, the survey was spread over companies and universities. This snowball method turns out to be quite effective, due to the efficiency in email services and the flexibility of a web survey.

\section{Data Analysis}

In total 100 respondents from the Netherlands and 98 respondents from Japan filled in the survey. Table 1 shows the general characteristics of the respondents. For both countries the majority of the respondents are between 18 and 39 of age, and students in profession. There is no professional driver respondent from Japan. The variation in profession is higher in the Netherlands than in Japan.

Between the respondents from these two countries, significant differences are observed in terms of car

Table 1 Characteristics of respondents

\begin{tabular}{|c|c|c|c|}
\hline Characteristics & & Dutch & Japanese \\
\hline \multirow[t]{4}{*}{ Age } & $18-24$ & $48 \%$ & $40.6 \%$ \\
\hline & $25-39$ & $36 \%$ & $38.5 \%$ \\
\hline & $40-64$ & $13 \%$ & $19.8 \%$ \\
\hline & 65 and older & $3 \%$ & $1.0 \%$ \\
\hline \multirow[t]{4}{*}{ Profession } & Student & $39 \%$ & $53.1 \%$ \\
\hline & Office worker & $28 \%$ & $30.2 \%$ \\
\hline & Professional driver & $5 \%$ & $0.0 \%$ \\
\hline & Other & $28 \%$ & $16.7 \%$ \\
\hline \multirow[t]{4}{*}{ Car possession } & None & $32 \%$ & $44.8 \%$ \\
\hline & 1 & $38 \%$ & $45.8 \%$ \\
\hline & $2-3$ & $28 \%$ & $9.4 \%$ \\
\hline & More than 3 & $2 \%$ & $0.0 \%$ \\
\hline \multirow[t]{6}{*}{ Annual mileage } & $0-1.000 \mathrm{~km}$ & $11 \%$ & $41.7 \%$ \\
\hline & $1.000-5.000 \mathrm{~km}$ & $19 \%$ & $29.2 \%$ \\
\hline & $5.000-10.000 \mathrm{~km}$ & $13 \%$ & $14.6 \%$ \\
\hline & $10.000-20.000 \mathrm{~km}$ & $29 \%$ & $12.5 \%$ \\
\hline & $20.000-30.000 \mathrm{~km}$ & $13 \%$ & $1.0 \%$ \\
\hline & More than $30.000 \mathrm{~km}$ & $15 \%$ & $1.0 \%$ \\
\hline \multirow[t]{4}{*}{ Primary trip purpose } & Commuting & $41 \%$ & $15.6 \%$ \\
\hline & Work related & $13 \%$ & $3.1 \%$ \\
\hline & Social \& Recreational & $45 \%$ & $56.3 \%$ \\
\hline & Shopping & $1 \%$ & $25.0 \%$ \\
\hline
\end{tabular}


possession, annual average mileage and primary trip purpose. Some Dutch drivers possess more than one car, while most of the Japanese drivers surveyed have one car or no car. Dutch respondents have a higher average mileage, make more frequent use of the car, and are more likely to commute by car. On the contrary, Japanese respondents have a lower mileage, use the car occasionally and mainly for private situations.

These differences are attributed to the different demographics in these two countries. Urban population density is higher in Japan than in the Netherlands. Ridership of public transport is also much higher in Japan. Using car to commute is far less popular in Japan than in the Netherlands. Japanese drivers often use the car for more private purposes, such as social and recreational activities.

The differences are also partially attributed to the unbalanced representation of the population within the respondents. As noted above, young people and students make out a big proportion of the respondents. The group of the respondents surveyed in this study is therefore an unrepresentative sample of the society. This bias will be taken into account in our analysis below.

\subsection{Information Priority}

Instead of categorizing drivers by their personal characteristics and then examining per group the preferred information, we have done the analysis in reverse. In the survey the respondents are asked to select the most relevant information for them to receive in order to change their behavior; they had to rank four types of information by priority. The results provide the reference point in our analysis: this priority information defines the respondent's character and attitude towards environment. By correlating this character to his driving style and experience with ITS, suitable Green ITS based on the preferred information can be designed.

Table 2 shows the frequencies of the four types of information being ranked with the highest priority. For both countries, money related information is the most important to drivers. The second most preferable information is about fuel consumption; this information is more preferable for Japanese drivers than for Dutch drivers. A remarkable

Table 2 Distributions of priority information

\begin{tabular}{lcc}
\hline Priority information & Dutch & Japanese \\
\hline Fuel consumption & $17 \%$ & $35 \%$ \\
Money saving & $64 \%$ & $56 \%$ \\
$\mathrm{CO}_{2}$ emission & $11 \%$ & $2 \%$ \\
Contribution to environment & $8 \%$ & $7 \%$ \\
\hline
\end{tabular}

difference is the importance of $\mathrm{CO}_{2}$ emission between the two countries.

Personal Character Several key factors have been chosen to uncover correlations between the preferred information and the character of the driver. To define the personal character of the driver, three key factors have been analyzed: age, profession and car possession. Age and profession tell something about the driver's daily life. Generally, office workers drive or commute more by car than students, which makes them a more important target for Green ITS. Car possession indicates the driver's need of a car and/or desire to drive. These factors are then compared with the preference of information.

The survey results show that Dutch respondents between the age 18 and 24, being students, and without car possession, are much more interested in money related information than other driver groups. The fact that office workers are forced to use the car and usually can declare their expenses to their employer explains the importance of fuel consumption instead of expenses. For the Japanese respondents, no correlation between choice of priority information and personal character has been identified. Each driver group (by age, profession or car possession) exhibits a distribution of priority information similar to the overall distribution in Table 2.

Driving Style Driving style characterizes the driver's driving behavior in daily life. The annual mileage and driving frequency provides information on car use. Drivers with a higher mileage produce more emissions. Primary trip purpose reveals the driver's familiarity with the routes during an ordinary trip. Commuting is often via the same route, meaning that the driver is familiar with the route and more likely to drive in a smooth way. This leads to less emission compared to recreational trips, where the driver is unfamiliar with the routes and has to learn about the conditions on these routes.

The survey results show that Dutch respondents with an average to low annual mileage (1,000 km to $10,000 \mathrm{~km})$ and less frequent use of car (1 to 8 times per month) are much more interested in money savings than other driver groups. No major difference has been identified between trip purposes. For the Japanese respondents, it appears that annual mileage and driving frequency have very little influence on the choice of priority information. However, respondents with their primary trip purpose as private (social recreational and shopping) are slightly less interested in money savings than other driver groups.

Experience with ITS Drivers' previous experience and familiarity with ITS systems and services make it easier for them to adapt to Green ITS, if the Green ITS is designed 
in a comprehensible way and in accordance with other ITS systems. In this survey, respondents are to rate their familiarity with cruise control, adaptive cruise control, lane departure warning, navigation systems, and intelligent information systems.

The survey results show a striking difference between the two countries in terms of the respondent's familiarity with ITS systems. Japanese respondents appear to be significantly less familiar with current ITS systems than the Dutch respondents, possibly due to the large amount of students in the respondent group.

Nonetheless, cruise control and navigation systems are widely used and perceived as familiar in both countries. Therefore a comprehensible new system should have the same level of adaptability as these two systems. For example, some interface aspects of these systems can be borrowed to maintain familiarity with the new system.

However, familiarity with ITS is shown to be not strongly related to the choice of priority information. Among drivers who are experienced with a particular ITS system, similar ratios in information preference are observed as those in Table 2.

Attitude Towards Environment Environmentally friendly behavior concerns actions that people take to improve the environment, such as recycling or low energy use. Most respondents hesitate to call themselves very environmentally friendly. The majority of respondents reflect on themselves as having an average personality towards environmental friendliness. Dutch respondents with $\mathrm{CO}_{2}$ emission and contribution to environment as their priority information do think that they are more environmentally friendly. On the contrary, Japanese respondents who are interested in information on their contribution to environment mostly refer to themselves as not environmentally friendly.

The respondents are also asked to reflect on their own fuel consumption behavior during driving. Respondents who choose contribution to environment as their priority information generally consider their driving behavior as not very fuel efficient, indicating their need to acquire such information. On the other hand, respondents who consider themselves as fuel efficient mainly choose fuel consumption as their priority information, signifying their desire to further reduce their fuel consumption.

Another question in the survey is about the influence of fuel price on car use. The intention of raising fuel price is to reduce fuel consumption. It is expected that respondents with fuel consumption and money savings as their priority information would be highly affected by fuel price. However, this is not the case in the survey. For the Japanese respondents, the effect of raising fuel price is independent of the driver's priority information; for each of the four respondent groups (as defined by Table 2), about half of the respondents report fuel price as having an influence while the other half state no influence. For the Dutch respondents, raising fuel price has limited influence on their behavior. For those with money savings as their priority information, the majority of them actually claim that fuel price has no influence at all on their behavior. A possible explanation is that these drivers are dependent on their car for commuting and cannot reduce their frequency of car use even under high fuel prices.

\subsection{Relevant Information for Changing Driving Behavior}

In the survey respondents are asked to rate several types of Green ITS information in terms of relevance. A high relevancy is interpreted as a high willingness to use Green ITS and to adapt one's driving behavior according to the received information. Information with the highest relevancy is the most preferable for the drivers to receive. Next to the types of information, respondents are also asked to indicate their preferred timing of receiving the information (discussed in section 4.3).

Monetary Impact The majority of drivers chose money related information as the most relevant motive for them to change their behavior. These drivers also include the most frequent drivers, i.e. those who produce the most emissions and with whom the most impact of Green ITS can be achieved. The information can be directly or indirectly related to money, including fuel consumption, money converted from fuel consumption, and the amount of money saved with fuel efficient behavior (Fig. 1). This type of information is much more relevant for Japanese drivers than for Dutch drivers, although more Dutch respondents have chosen money related information as their priority information than Japanese respondents.

Environmental Impact As for the types of environment related information, the indicator for fuel efficiency is the most popular among both Dutch and Japanese drivers (Fig. 2). For Dutch drivers, information on $\mathrm{CO}_{2}$ emission and contribution to environment are in general irrelevant, although the number of drivers who do indeed rate such information as relevant is not negligible. However, Japanese drivers generally find environment related information as relevant and are willing to receive such information. A possible reason is curiosity, i.e. the eagerness among Japanese drivers to use new technology and to receive all kinds of information.

Personal Driving Behavior Five elements of personal driving behavior are considered: speed, gear shift, 
Fig. 1 Level of relevance for money related information: Dutch drivers (left) and Japanese drivers (right)

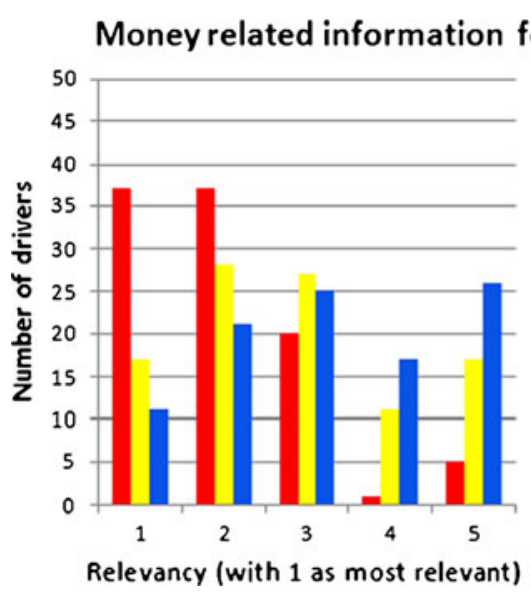

acceleration, deceleration, and route choice. Information related to such behavior includes the advice on optimal behavior, the en route reporting of driving progress, and the post trip reporting of driving history. Both Dutch and Japanese drivers find advices on their behavior very relevant (Fig. 3). Moreover, Japanese drivers show an equal interest in receiving information on their driving progress and history, whereas Dutch drivers find such information less interesting than advices. In terms of the behavioral elements, Dutch drivers prefer to receive information on speed and gear shift than on acceleration and deceleration, while Japanese drivers are indifferent.

\subsection{Timing of Information}

In the design of Green ITS, timing of the information supply is very important. Wrong timing of information can reduce the impact on driving behavior. An example is when advice on behavior is only given after trips. After trips drivers usually do not care about their behavior anymore and for the next trip they are more likely to have forgotten the advice.
In the survey respondents are presented with controlrelated advices and money-related information. They are then asked for their preferences on the timing of these advices and information. In terms of control-related advices, both Dutch and Japanese drivers prefer to receive such information during the trip; this preference is especially strong among Dutch drivers. In terms of money-related information (Fig. 4), Dutch drivers prefer to receive information on fuel consumption during the trip and information on money savings after the trip. Japanese drivers, however, prefer to receive both types of information during the trip.

\section{Proposal for Green its Design}

Based on results of the internet survey, improvements can be made to current Green ITS systems to better accommodate drivers' preferences. Accordingly, a new Green ITS design is proposed here.

Data Flow History, progress and advice on driver behavior are based on the personal driving behavior, i.e. the factors
Fig. 2 Level of relevance for environment related information: Dutch drivers (left) and Japanese drivers (right)

\section{Environment related information for Dutch (left) and Japanese drivers}
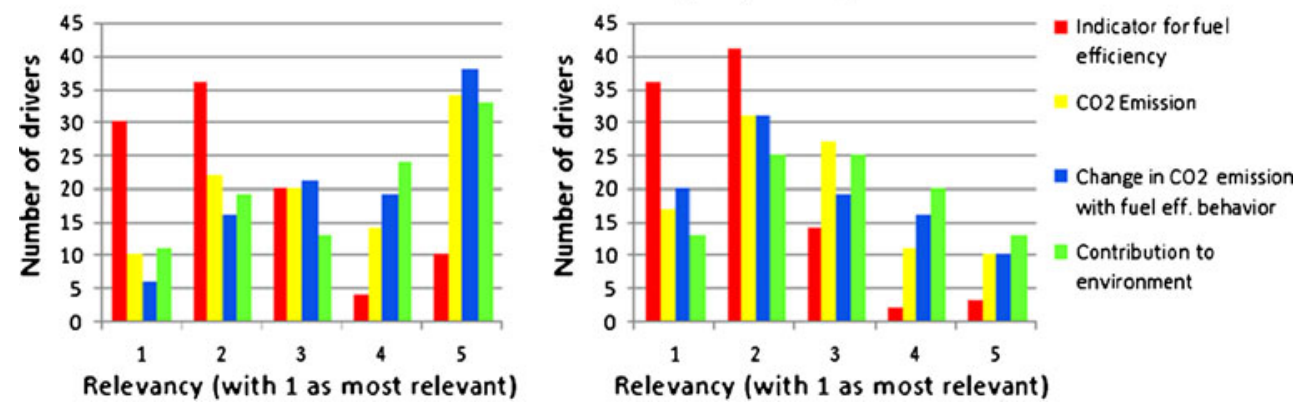
Fig. 3 Level of relevance for different types of Green ITS information: Dutch drivers (left) and Japanese drivers (right)

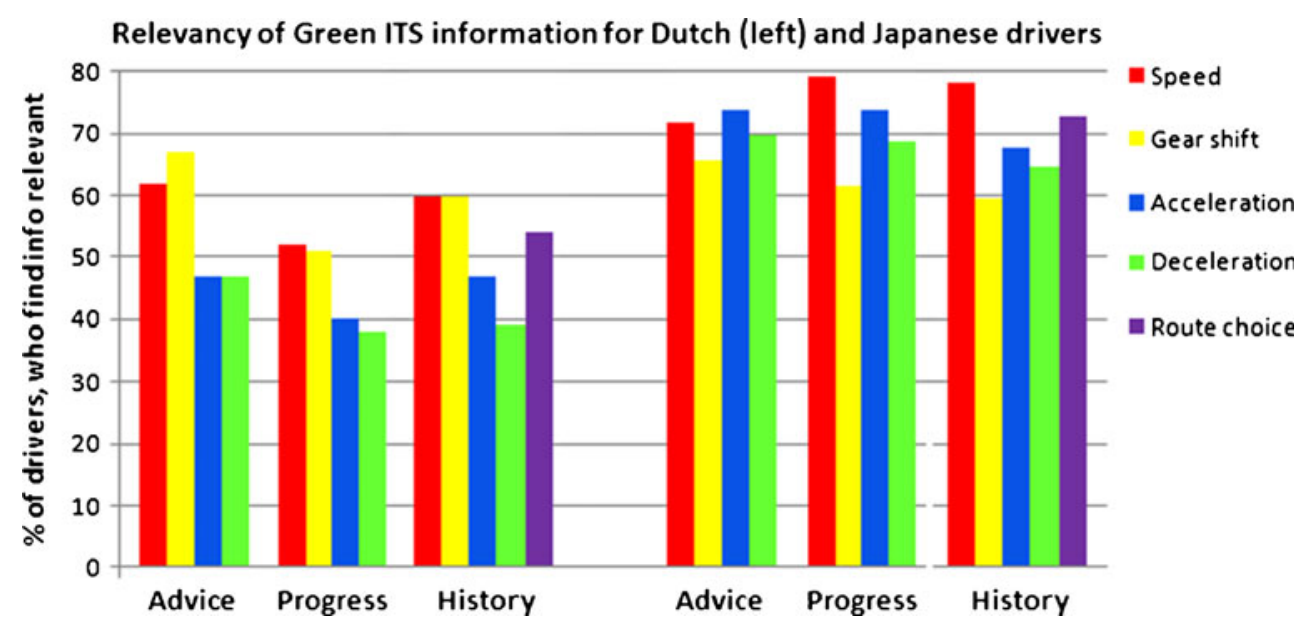

of speed, gear shift, and ac-/deceleration. During the trip, data of these factors are collected and then compared to the optimal fuel efficient behavior (e.g. optimal speed, optimal gear shift moment) in order to determine the advice to be given to the driver. Once the driver is following the advice, the difference due to this behavioral change is calculated.

Besides information on personal driving behavior, other types of information are also available to the driver. Money related information includes fuel consumption in liters per kilometer, fuel consumption calculated in monetary values, and the amount of money savings in fuel consumption (compared to the "previous" behavior). Environment related information includes the amount of $\mathrm{CO}_{2}$ emission and the reduction of $\mathrm{CO}_{2}$ emission.

Type of Information The use of Green ITS should not affect the driver's safety. To avoid stress, distraction and confusion during driving, drivers can choose to only display the essential information directly related to their driving behavior such as progress of the personal driving behavior. Other types of information can be displayed before and/or after the trip. Information that has direct effects on the driving behavior is advice for fuel efficient behavior. Unlike information about driving progress and history, advice can be directly adapted by the driver. The driver has the choice to display advice for speed, gear shifts, and ac-/deceleration.

For money related information, it is suggested to only display fuel consumption during the trip. Because of changing fuel prices, monetary values are not always accurate and are better displayed before or after the trip (with the driver being able to specify the fuel price). All environment-related information can be made available after the trip.

User Interface Figure 5 gives an impression on the proposed Green ITS system. During the trip the information displayed should be easily understandable and without any confusion or distraction. An on board unit is attached to the dashboard with its screen showing information on gear shift, and ac-/deceleration. The screen will gradually turn red when inefficient behavior is detected, such as excessive pedal pressure and gear shift at high revolutions. The deeper the color, the more inefficient the behavior is. With fuel efficient behavior the display turns into green again. The bar in the corner displays how much fuel is left. The factors of speed,
Fig. 4 Preferred timing of the money related information: Dutch drivers (left) and Japanese drivers (right)

\section{Timing of money related information for Dutch (left) and Japanese drivers}
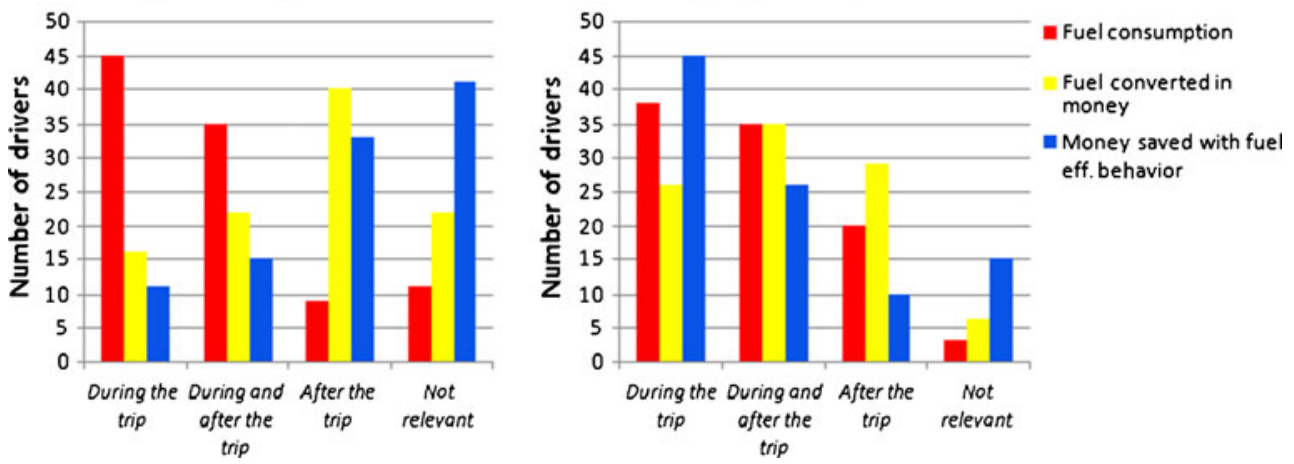

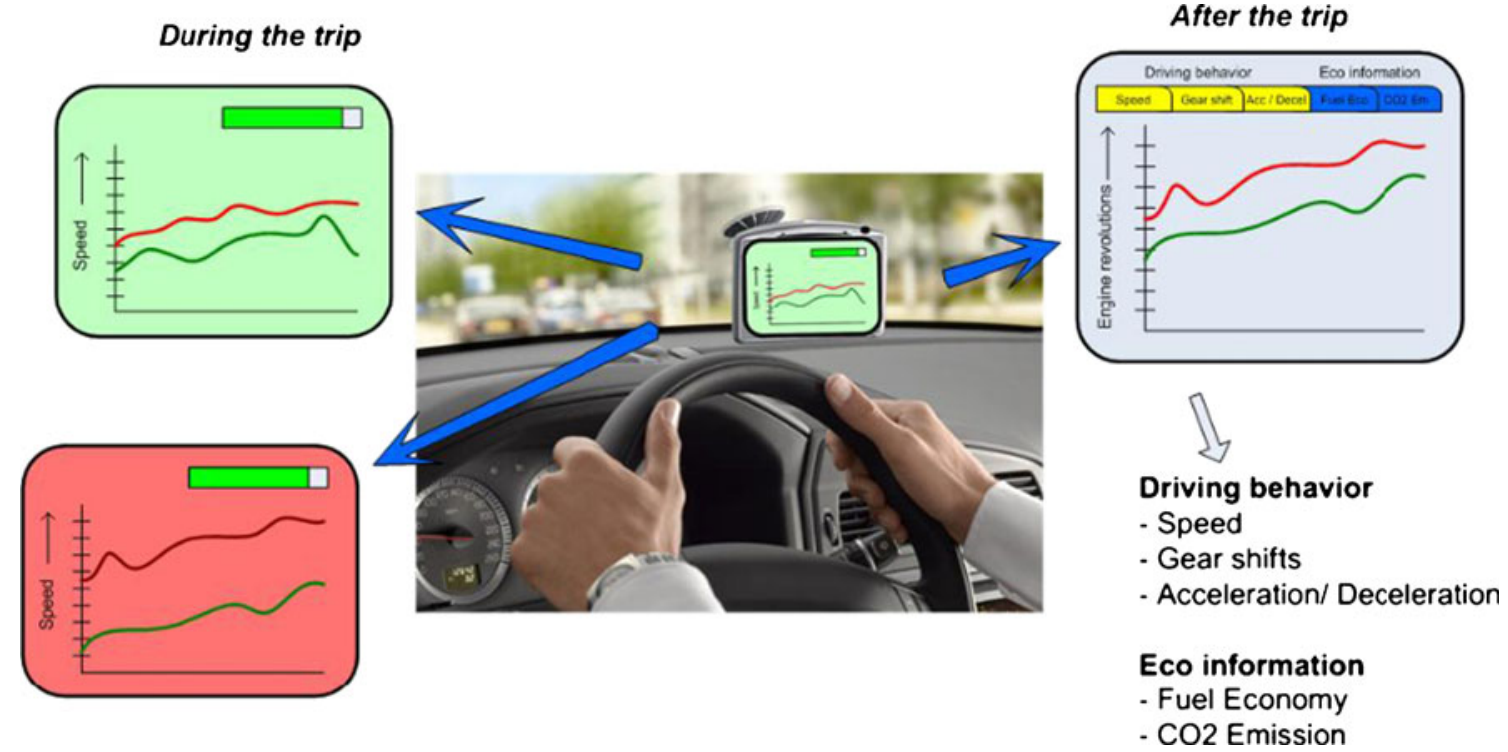

Fig. 5 Conceptual sketch of the proposed Green ITS system

gear shift, and ac-/deceleration are displayed in line charts on screen, showing both the actual behavior (the red curve) and the optimal behavior (the green curve).

Graphs displayed after the trip provide overviews on the differences between the actual fuel consumption and the fuel consumption under fuel efficient behavior (in both volume and money). This tells the driver how much excessive fuel has been consumed and how much money could have been saved with fuel efficient behavior. The same concept applies to environment related information, i.e. differences between the actual $\mathrm{CO}_{2}$ emission and the emission under optimal behavior.

\section{Conclusions and Discussions}

Through an internet survey we studied the attitude towards Green ITS among drivers in the Netherlands and Japan. It has been shown that the biggest motivation for drivers to follow fuel efficient behavior is the potential money savings. Green ITS should strive to provide drivers with information on how much money they would save. Next to that, drivers are also interested in information on fuel consumption during their trips. The least preferred information is actually about the impact on environment, such as $\mathrm{CO}_{2}$ emission. The cause of this disinterest could be that drivers do not see a direct relationship between their car use and its impact on the environment. In order to make these drivers aware of their impact on environment and to motivate them to adapt to environmentally friendly behavior, we need to help the driver establish a direct, understandable and imaginable relationship between their behavior and the environmental impact. An example could be to make a comparison between emission and the reduction of trees.

Personal characteristics, experience with ITS, and attitude towards environment have been shown to cast only a minor influence on the driver's preference of information in Green ITS. Correlations, however, have been found between the preference of information and the driving style. Overall, the majority of respondents consider information provided in Green ITS as relevant and they are willing to use such systems. This positive attitude is expected to contribute to high acceptance and frequent use of Green ITS. However, the limited scope of this study should be noted in future user preference studies where more representative respondent sampling should be made. It should also be noted that drivers' actual preference and behavior (revealed preference) may turn out to be different than what they have stated in the questionnaire survey (stated preference).

Green ITS is a relatively new concept. Very limited deployment experiences have been reported in literature. For future implementation and promotion of Green ITS, information on driver experiences should be gathered. By analyzing these experiences, likes and dislikes of users can be identified, which will make room for further improvement. These improvements can be based on the type of information supply, the timing of information supply and the display of information. Different types of information or modes of display are expected to have different effects on the driving behavior.

In the design of Green ITS, safety, distraction and other psychological influences on the driver are of great importance. In the proposed design, the system provides information on driving behavior, expenses on fuel consumption and environmental factors. During the trip, advice and fuel consumption 
will be displayed with simple indications on an on-board screen. After the trip other information will be presented with trends, numbers and texts.

Acknowledgments The research described in this paper was conducted during the first author's bachelor assignment at Toyota InfoTechnology Center Co., Ltd. The authors would like to thank Sachi Mizobuchi and the Department of Research and Development for their enthusiasm and contribution to this research. The authors also acknowledge the constructive comments of an anonymous referee.

Open Access This article is distributed under the terms of the Creative Commons Attribution Noncommercial License which permits any noncommercial use, distribution, and reproduction in any medium, provided the original author(s) and source are credited.

\section{References}

1. UNFCCC, Kyoto Protocol. Available online: http://unfecc.int/ kyoto_protocol/items/2830.php (1997)

2. Logghe, S., Van Herbruggen, B., Van Zeebroeck, B.: Emissions of Road Traffic in Belgium. Transport \& Mobility Leuven (2006)

3. Fergusson, M., Smokers, R., Passier, G., ten Brink, P., Watkins, E., Valsecchi, C., Hensema, A.: Possible regulatory approaches to reducing CO2 emissions from cars. European Commission, Brussels (2007)

4. Bell, M.C.: Environmental factors in intelligent transport systems. IEE Proceedings: Intelligent Transport Systems 153, 113-128 (2006)

5. FIAT, eco:Drive. Available online: http://www.fiat.co.uk/ecodrive/ (2009)

6. van Driel, C.J.G.: Driver Support in Congestions. TRAIL Research School, Delft (2007)

7. van Breugel, P.B., Baum, A., Calovi, L., Hackman, M., de Gier, C. W., Juneholm, M., Klaeboe, R., Pucher, E., Kampfer, A., Vinot, J.: Examples of air quality measures near roads within Europe: National measures of the international CEDR air quality group. Rijkswaterstaat, Delft (2005)

8. van Maarseveen, M.F.A.M., van der Voort, M.C., van Driel, C.J.G.: Qualitative Survey on Fuel Economy Devices. University of Twente, Enschede (2002)

9. de Argaez, E., Internet World Stats. Available online: http://www. internetworldstats.com/ (2011)

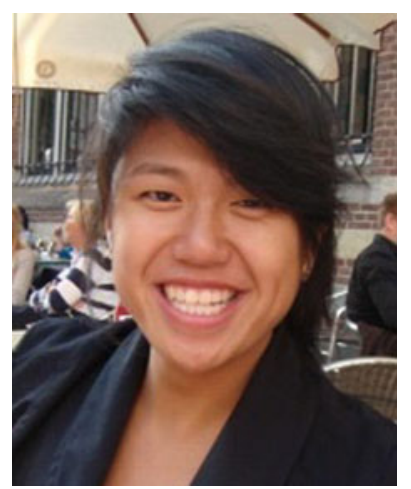

Wing Yan Man is a postgraduate student at the University of Twente, Enschede, the Netherlands. Currently she is working towards her Master's degree in Civil Engineering.

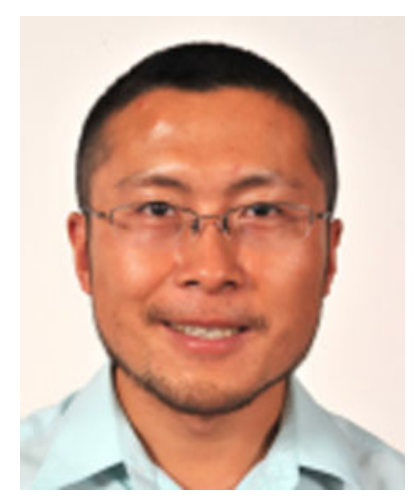

Jing Bie is an assistant professor in the University of Twente, Enschede, the Netherlands. He holds a Ph.D. degree (2008) from the Hong Kong University of Science and Technology.

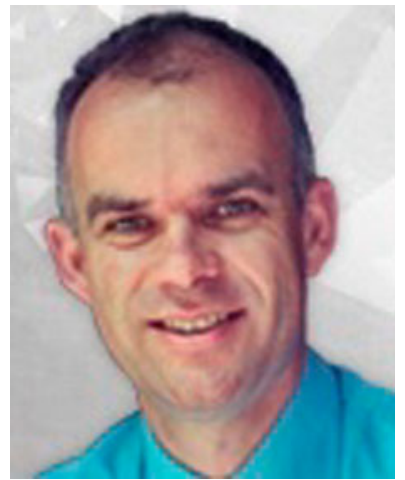

Bart van Arem is a professor in Delft University of Technology, Delft, the Netherlands. He holds a Master's degree (1986) and a Ph.D. degree (1990) from the University of Twente. 